Article

\title{
A Multi-mode SAR Imaging Chip based on a Dynamically Reconfigurable SoC Architecture Consisting of Dual-operation Engines and Multilayer Switching Network
}

\author{
Teng Long, Zhu Yang, Bingyi Li, Liang Chen", Zegang Ding, He Chen, Yizhuang Xie \\ Beijing Key Laboratory of Embedded Real-time Information Processing Technology, Beijing Institute of \\ Technology, Beijing 100081, China; longteng@bit.edu.cn(T.L); yangzhu@bit.edu.cn(Z.Y); \\ libingyi_bit@bit.edu.cn(B.L.); z.ding@bit.edu.cn(Z.D); chenhe@bit.edu.cn(H.C); xyz551_bit@bit.edu.cn (Y.X.). \\ * Correspondence: chenl@bit.edu.cn; Tel.: +86-6842-9855
}

\begin{abstract}
With the development of satellite load technology and very-large-scale integrated (VLSI) circuit technology, on-board real-time synthetic aperture radar (SAR) imaging systems have facilitated rapid response to disasters. Limited by severe size, weight, and power consumption constraints, a key challenge of on-board SAR imaging system design is to achieve high real-time processing performance. In addition, with the rise of multi-mode SAR applications, the reconfiguration of the on-board processing system is beginning to receive widespread attention. This paper presents a multi-mode SAR imaging chip with SoC architecture based on the reconfigurable double-operation engines and multilayer switching network. We decompose the commonly used extend chirp scaling (CS) SAR imaging algorithm into 8 types of double-operation engines according to the computing orders, and design a threelevel switching network to connect these engines for data transition. The CPU is responsible for engine scheduling based on data flow driven with instructions to implement each part of the CS algorithm. Thus, multi-mode floating-point SAR imaging processing can be integrated into a single Application-Specific Integrated Circuit (ASIC) chip instead of relying on distributed technologies. As a proof of concept, a prototype measurement system with chipincluded board is implemented, and the performance of the proposed design is demonstrated on Chinese Gaofen-3 stripmap continuous imaging. A chip requires 9.2 s, $50.6 \mathrm{~s}$ and $7.4 \mathrm{~s}$ for a stripmap with $16,384 \times 16,384$ granularity, multi-channel stripmap with $65.536 \times 8192$ granularity and multi-channel scan mode with $32,768 \times 4096$ granularity and $6.9 \mathrm{~W}$ for the system hardware to process the SAR raw data.
\end{abstract}

Keywords: synthetic aperture radar (SAR); real-time processing; single FPGA node imaging processing; multi-node parallel accelerating technique

\section{Introduction}

Synthetic aperture radar (SAR) is a kind of active-observation system to the Earth, which is able to work day and night under all weather conditions. As an important technique for spaceto-earth observation, spaceborne synthetic aperture radar (SAR) has the ability to collect data continuously over large areas at high resolution, making it a flexible and effective tool for information retrieval[1]. SAR plays an important role in disaster emergency response, environmental monitoring, resource exploration, and geographic information access[2-4] . Recent publications have reviewed the applications of satellite remote sensing techniques for hazards manifested by solid earth processes, including earthquakes, volcanoes, floods, 
landslides, and coastal inundation[5-7]. Therefore, the development and research on SAR attract more and more attention of the countries around the world, such as system design, raw data simulation, imaging algorithm, information extraction and so on.

So far, various countries have launched a large number of SAR satellites such as Sentinel1[8], TanDEM-X/TerraSAR-X (TDX/TSX)[9], ALOS[10] and Chinese Gaofen-3[11]. Most of the above-mentioned missions impose high demands on the real-time performance of SAR data processing. On-board processing is an efficient solution that allows higher precision SAR data to be processed, leading to better image quality and enabling optional image compression. With these processed data products, decision makers can quickly plan and respond. Normally, spaceborne real-time processing performance improvement mainly includes three aspects, optimizing appropriate algorithm, developing an optimal algorithm implementation strategy and building a high-performance processing platform.

In recent years, Chirp Scaling (CS) algorithm has become the mainstream in SAR imaging field, especially for spaceborne SAR. It consists basically of multiplying the SAR data in the range-Doppler domain with a quadratic phase function (chirp scaling) in order to equalize the range cell migration to a reference range, followed by a range compression and SRC in the wavenumber domain. Although the SRC is strictly correct only for one reference range, it is updated as a function of the azimuth frequency. The processing proceeds with phase multiplies and FFT operations, which make the algorithm extremely efficient. Besides, CS algorithm can significantly improve the performance of the SAR imaging because of the accurate processing in the squint mode[12]. Due to the advantage of high efficiency, CS and its improved algorithms ,mainly include Nonlinear Chirp Scaling(NCS)[13], Extend Chirp Scaling (ECS)[14] and so on, are still employed for spaceborne SAR imaging of various modes, including Stripmap mode[15], Spotlight mode[16], Scan mode[17], multi-channel mode[18] and TOPS mode[19]. This paper chose the ECS for multi-mode SAR imaging algorithm. With the development of SAR technologies, there is a higher demand for the resolution and swath of SAR images, which brings a huge amount of imaging calculation. Since the amount of computation of the algorithm does not change substantially (depending on the size and number of FFTs), algorithm engineering optimization is in fact indispensable for the fast and even real-time imaging processing in spaceborne system.

As early as 2000, the MIT Lincoln Laboratory began a study of the implementation of realtime signal processors for SAR front-end signal processing[20]. The processors were designed, based on their own VLSI bit-level systolic array technology, to have high computational throughput and low power implementations. S. Langemeyer et al. of the University of Hannover, Germany, proposed a multi-DSP system for real-time SAR processing using the highly parallel digital signal processor (HiPAR-DSP) technique in 2003[21]. The small volume and low power consumption of their processor make it suitable for on-board usage in compact air- or spaceborne systems. The Jet Propulsion Laboratory (JPL) has also worked to develop on-board processing. An experimental SAR processing system based on VLSI/SOC hardware has been proposed[22]. A fault-tolerant FPGA (Xilinx Virtex-II Pro)-based architecture has been proposed and tested using the SIR-C data[23, 24]. The University of Florida developed a high-performance space computing framework based on a hardware/software interface in 2006[25]. An FPGA serves as the co-processor/accelerator of the CPU. A near-real-time SAR processor (NRTP) was developed by the Indian Space Research Organization (IRSO) based on the Analog Devices TigerSHARC TS101S/TS201S DSP multiprocessor. On-board or on-ground quick-look real-time SAR signal processing was found to be achievable for ISRO's RISAT-1[26]. With the rapid increase in the storage and computing capacities of commercial-off-the-shelf (COTS) FPGAs, the state-of-the-art Xilinx Virtex-6 FPGA was adopted for an entire real-time SAR imaging system in 2013[27]. In recent years, graphics processing units (GPUs), with their high computing power, have also been used for real-time SAR processing[12]. 
3 of 16

Building a high-performance SAR real-time processing platform for space deployment is hampered by the hostile environmental conditions and power constraints in space. The FPGA, ASIC, DSP, CPU, and GPU are, to some extent such as SoPC, superior with respect to real-time processing. Although the GPU has a high processing power, its large power consumption and low radiation resistance makes it unsuitable for the harsh conditions of spaceborne on-board processing. The CPU and DSP take advantage of their design flexibility by software reconfiguration, however, they cannot provide sufficient FLOPS per watt, which leads to a bottleneck in large-scale and high-resolution applications. Benefiting from its customized design, FPGAs and ASICs can provide sufficient processing power and high computation ability; however, in implementing an FPGA or ASIC for Specific-mode SAR imaging, the large-scale, complicated logic design requires a longer development period.

Our preliminary work[28, 29] described two options for the SAR imaging system: FPGA+ASIC and single FPGA integration. Especially for the second option, we propose a multinode parallel accelerating system to realize an on-board real-time SAR processing system. However, all systems focus on the standard stripmap, which is the most foundational mode of SAR imaging application. The other reference[30] focuses on analyzing the methodology of CS coefficient decomposition and implementation in FPGAs, not ASIC chips. In this paper, to address the increasing need for wide-breadth imaging, multi-channel correlated modes must be considered. This paper presents a multi-mode SAR imaging chip with SoC architecture based on reconfigurable double-operation engines and a multilayer switching network. We provide the following contributions to the existing body of research:

- The first integrated multi-mode SAR imaging system on a single ASIC chip for spaceborne application is presented. The indicators of the chip, such as performance, area, and power, can satisfy the needs of spaceborne SAR imaging processing.

- According to the operational characteristics of the algorithm, a double-operation enginebased mapping strategy is developed and described in detail. A total of 8 types of engines are mounted in a multilayer switching network, and the whole imaging procedure can be implemented in a data-stream-driven mode.

The remainder of the paper is organized as follows: Section 2 reviews the CS algorithm and describes the double-operation engine mapping strategy. Section 3 presents a single-chip integration design for optimizing the CS algorithm implementation with the multilayer switching network. In Section 4, the corresponding hardware realization details and results are discussed. A comparison with related work is conducted to demonstrate the validity of our system. Section 5 concludes the paper.

\section{Chirp Scaling (CS) Algorithm Review and double-operation engine-based mapping strategy}

\section{1 Extend Chirp Scaling (ECS) Algorithm Review}

The CS algorithm is one of the most fundamental and popular algorithms for spaceborne SAR data processing. Compared to other algorithms, the advantage of the CS algorithm lies primarily in its use of the "chirp scaling" principle, in which phase multiplies are used instead of a time-domain interpolator to implement range-variant range cell migration correction (RCMC) shift[31]. This algorithm can also solve the problem of the dependence of the secondary range compression (SRC) on the azimuthal frequency because of the requirement for data processing in the two-dimensional frequency domain. 
4 of 16

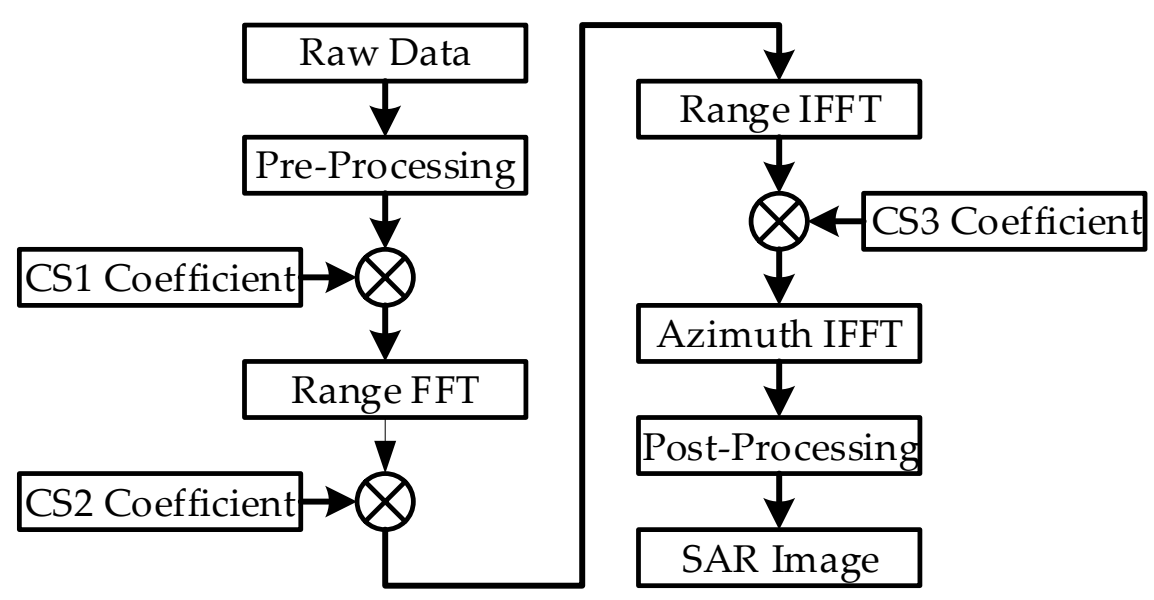

Figure 1. Flowchart of the chirp scaling (CS) algorithm.

The imaging algorithm illustrated in Figure 1 represents the heart of the integrated SAR imaging algorithm, which can incorporate stripemap, scan, multichannel and other extend modes. This paper focuses on standard- and multichannel-strip modes. Thus, the preprocessing mainly includes operations of inverse filtering and sub-channel data fusion[32]. Fast Fourier transformation (FFT)/inverse FFT (IFFT) and CS coefficient calculations are the major features of the main procedure of the algorithm, constituting $\sim 80 \%$ of the overall computation. The steps in the CS algorithm are as follows:

First, the SAR raw data after pre-processing are transferred to the Range-Doppler domain via a FFT in the azimuthal direction. Second, the data are multiplied by the CS1 Coefficient to achieve the chirp scaling, which makes all the range migration curves the same. The CS1 Coefficient can be described as:

$$
f_{1}\left(t, f_{h} ; r_{r e f}\right)=\exp \left[-j p b_{r}\left(f_{h} ; r_{r e f}\right) c_{s}\left(f_{h}\right)\left(t-\frac{2}{c} r_{r e f}\left(1+c_{s}\left(f_{h}\right)\right)\right)^{2}\right],
$$

where $t$ is the range time, $f_{h}$ is the azimuthal frequency, $r_{r e f}$ is the reference distance, $b_{r}\left(f_{h} ; r_{r e f}\right)$ is the modulating frequency in the phase center of the range direction, and $c_{s}\left(f_{h}\right)$ is the curvature factor, expressed as follows:

$$
\begin{gathered}
c_{s}\left(f_{h}\right)=\frac{\sin j_{r e f}}{\sqrt{1-\left(\frac{l f_{h}}{2 v}\right)^{2}}-1,} \\
b_{r}\left(f_{\eta}, r_{r e f}\right)=\frac{\left[1-\left(\frac{\lambda f_{\eta}}{2 v}\right)^{2}\right]^{\frac{3}{2}} \times b}{\left[1-\left(\frac{\lambda f_{\eta}}{2 v}\right)^{2}\right]^{\frac{3}{2}}+b r_{r e f} \sin \varphi_{r e f}\left(\frac{\lambda f_{\eta}}{2 v}\right)^{2}} .
\end{gathered}
$$

where $\lambda$ is the wave length, $b$ is the modulation frequency of the transmitted signal, $\varphi_{r e f}$ and $v$ represent equivalent squint angle and equivalent squint velocity, respectively. These variables can be described as follows:

$$
v=\sqrt{\frac{\lambda r f_{r}}{2}+\left(\frac{\lambda f_{d}}{2}\right)^{2}}
$$


5 of 16

where $f_{d}$ represents DFC and $f_{r}$ represents DFR. Because CS1 Coefficient and CS2 Coefficient consider the range dimension, the initial values obtained by the ephemeris parameter can be adopted to simplify calculation.

Third, the data are transferred to the two-dimensional frequency domain via an FFT in the range direction. Next, the data are multiplied by CS2 Coefficient to complete the range compression, the SRC, and the remaining RCMC. The CS2 Coefficient can be described as follows:

$$
f_{2}\left(f_{t}, f_{h} ; r_{r e f}\right)=\exp \left[-j p \frac{f_{t}^{2}}{b_{r}\left(f_{h} ; r_{r e f}\right)\left[1+c_{s}\left(f_{h}\right)\right]}\right] \exp \left[+j \frac{4 p}{c} f_{t} r_{r e f} c_{s}\left(f_{h}\right)\right]
$$

where $f_{t}$ is the range frequency.

Next, the data are transferred to the Range-Doppler domain via an inverse FFT in the range direction. The data can be multiplied by the CS3 Coefficient to complete the azimuth compression and the phase correction. The DFR based on the raw data is used to refine the equivalent velocity $v$ to ensure the precision of the 3rd phase function and is described as follows:

$$
f_{3}\left(t, f_{h}\right)=\exp \left[-j \frac{2 p}{l} c t\left(1-\sin j \text { ref } \sqrt{1-\left(\frac{l f_{h}}{2 v}\right)^{2}}\right)+j \frac{4 p}{c^{2}} b_{r}\left(f_{h} ; r_{r e f}\right)\left(1+c\left(f_{h}\right)\right) c\left(f_{h}\right)\left(r-r_{r e f}\right)^{2}\right] .
$$

Finally, the inverse FFT operation in the azimuthal direction is eOEcuted to complete the CS algorithm. A visualized grayscale image can be obtained after performing the 8-bit quantization operation, which can be consider as a kind of post-processing.

\section{2 double-operation engine-based mapping strategy}

Through analysis, we divide the process of calculating the CS coefficients into multiple operations, as shown in Table 1.

Table 1 The basic operations called by CS phase function estimation

\begin{tabular}{cccc}
\hline Stage & $\begin{array}{c}\text { Basic } \\
\text { operation }\end{array}$ & $\begin{array}{c}\text { Trigonometric } \\
\text { operation }\end{array}$ & $\begin{array}{c}\text { Transcendental } \\
\text { operation }\end{array}$ \\
\hline CS1 Coefficient & Add, subtract, multiplication, divide & $\begin{array}{c}\text { Sine, Cosine, } \\
\text { arctangent }\end{array}$ & Root, exponent \\
CS2 Coefficient & Add, subtract, multiplication & Sine, Cosine & Root, exponent \\
CS3 Coefficient & Add, subtract, multiplication & $\begin{array}{c}\text { Sine, Cosine, } \\
\text { arctangent }\end{array}$ & Root, exponent \\
\hline
\end{tabular}

Corresponding to the operation type, the implementation calls for the use of three types of engines, single-, dual- and triple-operator engines $(\mathrm{OE})$, which can be defined as follows:

- A single-operator engine involves two inputs and one output to perform a single type of calculation.

- A dual-operator engine has three inputs and one output to perform a combination of two types of calculations.

- A triple-operator engine has four inputs and one output to perform three types of calculations.

Taking the first phase function estimation as an example, the cost and characteristics of the CS algorithm using different combinations of operation types are shown in Table 2. Through a theoretical analysis of the different engines, we find that the dual-operator engine offers an optimal implementation by requiring the fewest engines, the smallest number of iteration cycles and the fewest input channel resources. Figure 4 shows an example of CS phase function calling 
6 of 16

by the dual-operator engine, which includes seven operations such as square root and addition, square root and multiplication, and multiplication and exponentiation.

Table 2 The overheads and characteristics of CS algorithm implemented by different operation combinations

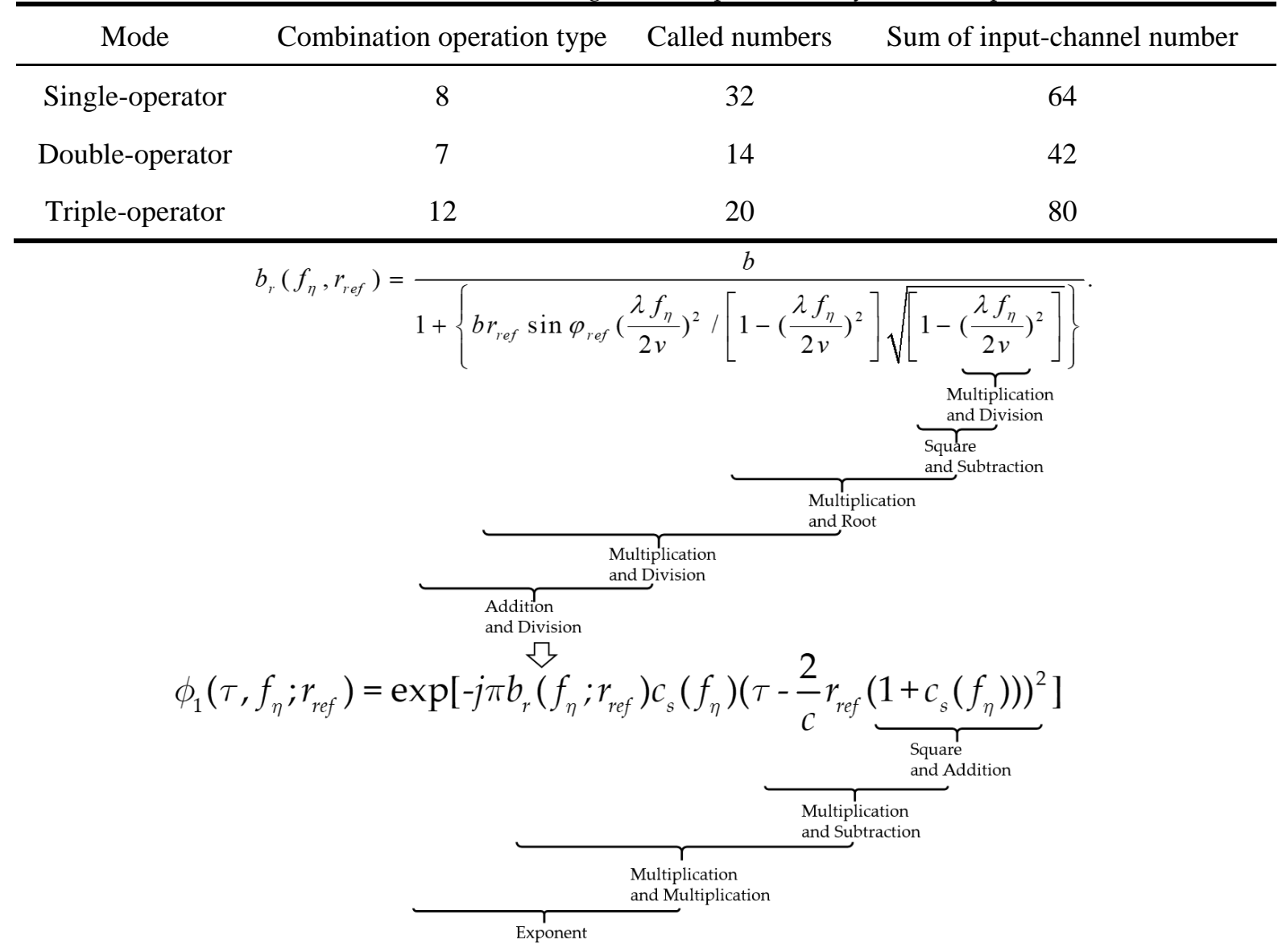

Figure 2 Double operators split instances $b_{r}$ and 1st phase function.

The design of this paper assumes that the FFT is also a basic operation; thus, "FFT- multiplication" can be regarded as one type of double operator in the switching network. Through comprehensive analysis, the CS algorithm needs 8 types of double-operator engines in total, shown in the following:

Table 2

\begin{tabular}{cc}
\hline Stage & Function \\
\hline OEFFT & FFT and Multiplication \\
OEME & Multiplication and Exponent \\
OEMA & Multiplication and Addition \\
OEMD & Multiplication and Division \\
OEMM & Tow Multiplication \\
OERM & Root and Multiplication \\
OERA & Root and Addition \\
OETD & Trigonometric and Division \\
\hline
\end{tabular}

The switching network based on the above eight dual-operator engines can not only implement various operations in the CS algorithm but also efficiently perform integrated SAR 
pre-processing and post-processing, thereby reducing the complexity of the system circuit. The hardware scheme design will be described in the following chapter.

\section{The CS imaging algorithm optimal scheme based on dual-operator engines}

As mentioned before, considering the integrated SAR imaging algorithm from the perspective of vector operations, it can be considered that the part with many FFT/IFFT operations is the key operation part of the imaging algorithm, and the complexity of the CS phase operation circuit is reduced by integrating the CS factor operation for different algorithm modes. Effort to improve the operational efficiency can improve the overall system performance. Our previous study proposed a switching network architecture based on the operator engine to implement CS phase factor operations as shown in Figure 3[30]. Among them, the computation engine undertakes the main computational task, all operator engines interconnect through the exchange network, the interconnection channel between the engines is composed of the node that exchanges the network, and the system finishes the factor operation through each operation engine in the dispatching exchange network. This paper focuses on how to design a suitable architecture of the above-mentioned 8 types of double-operator engines to reduce the complexity of the factor operation circuit and improve the efficiency and reusability of the calculation, as well as how to reasonably plan FFT/IFFT coordination and scheduling. Switching Matrix 0

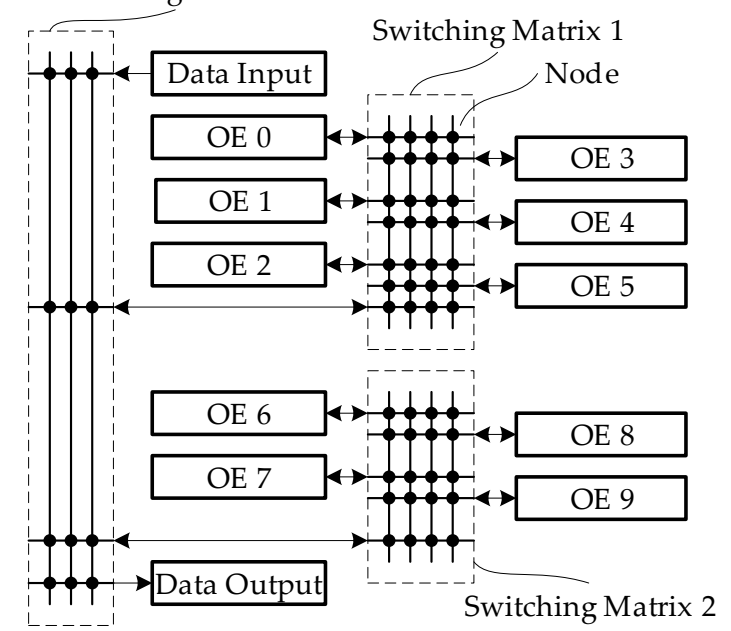

Figure 3. Operator engine and switching network.

\subsection{The design of engine unit}

Considering that the above-mentioned eight types of dual-operator engines should be attached to a uniform switch network, unified engine architectures with identical interface protocols are become more important. The topology structure of the engine unit designed according to this principle is shown in Figure 4. 


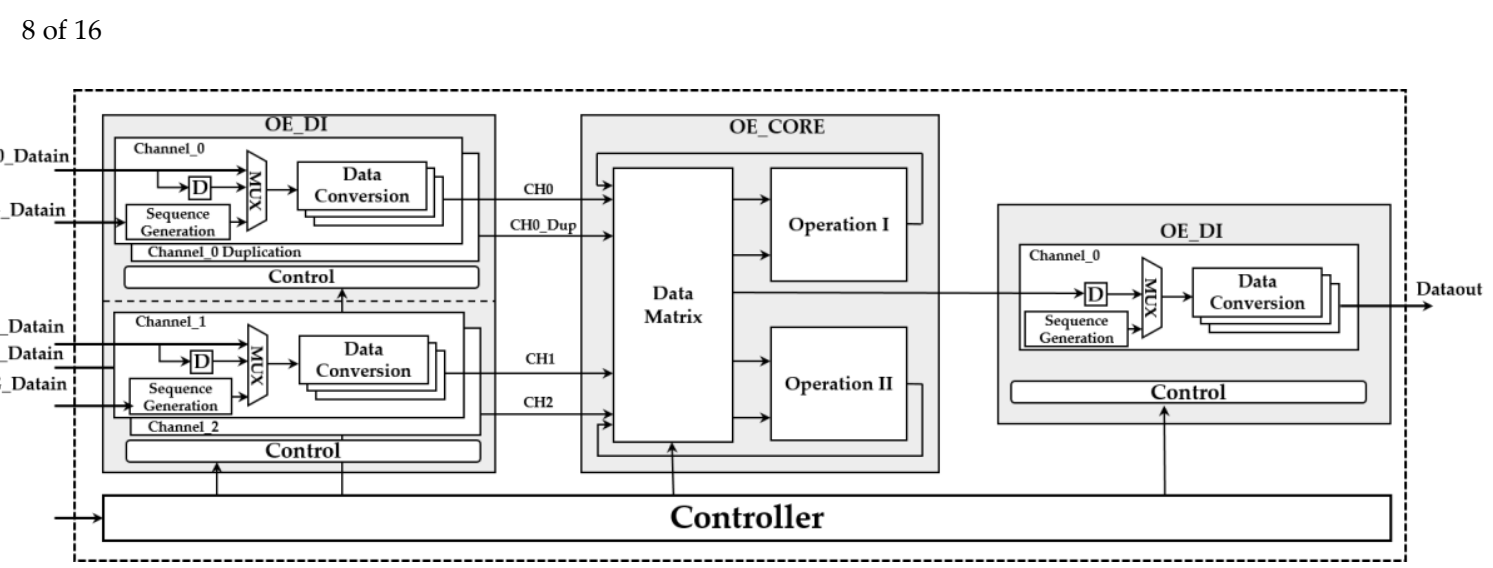

Figure 4. The topology structure of the engine unit.

The OE module is defined as an arithmetic engine module in which the data are three inputs and one output and is controlled as one input. The interior of OE is mainly composed of DI, CORE and DO.

OE_DI module: This module is mainly responsible for the pre-processing of the three input datasets before calculation to ensure that the data entering the calculation logic meets the timing and format requirements of different calculation modes. The control signal is responsible for the mode and parameter configuration of the module. The data selection section is appended with an additional sequence generation section, which is mainly for coordinate generation and correlation operations in the algorithm. The data conversion part can realize floating point data shifts (i.e., zoom in or out), finding negative and absolute values, and delay adjustment, and each function has a corresponding bypass path. The three input channel data processes are independent of each other, and the control section controls the output data synchronization. Simultaneously, we defined a channel-data replication operation path to handle the squared operations involved in the algorithm. Finally, the DI module will have 4 channels of data output to OE_CORE.

OE_CORE module: Each OE_core contains two two-input computing IPs that support the use of two IPs. The DATA_MATRIX part is responsible for data scheduling and grouping. By configuring the input data ID, the IP core can perform the calculation in the order of the algorithm. The configuration method is shown in the table. Because of inconsistent calculation delays of floating-point IP cores, DATA_MATRIX also needs to synchronize the output data.

Table 3 The ID list of DATA_MATRIX configuration

\begin{tabular}{cc}
\hline ID & Corresponding port \\
\hline 0x00 & Calculated output \\
0x01 & The first input of Operation I \\
0x02 & The second input of Operation I \\
0x03 & The first input of Operation II \\
0x04 & The second input of Operation II \\
\hline
\end{tabular}

OE_DO module: This module is mainly responsible for the post-processing of the calculation results. The internal structure of the module is the same as the OE_DI single channel. OE_DO outputs a single-channel result and can be used as the input for the next OE.

Controller module: This module is responsible for receiving the configuration information of the external control instruction, completing the reset of the entire module, parameter configuration, work mode selection, interrupt handling and work status monitoring. 
Based on the optimized data network processing architecture, to realize the multi-mode integrated spaceborne SAR real-time imaging processing flow, according to the imaging mode for selecting the corresponding data flow scheduling mode, we designed a multi-mode integrated spaceborne SAR real-time imaging processing system. The multi-mode integrated spaceborne SAR real-time imaging processing system is designed with a classic SoC structure. The bus and DMA architecture is used to build a data exchange network. The data stream drive mode is used to schedule each dual-operator engine to complete multi-mode integrated spaceborne SAR real-time imaging processing flow. The multi-mode integrated spaceborne SAR real-time imaging processing system is mainly composed of six parts: the CPU, data movement, arithmetic engine storage, high-speed interface, global logic and peripherals.

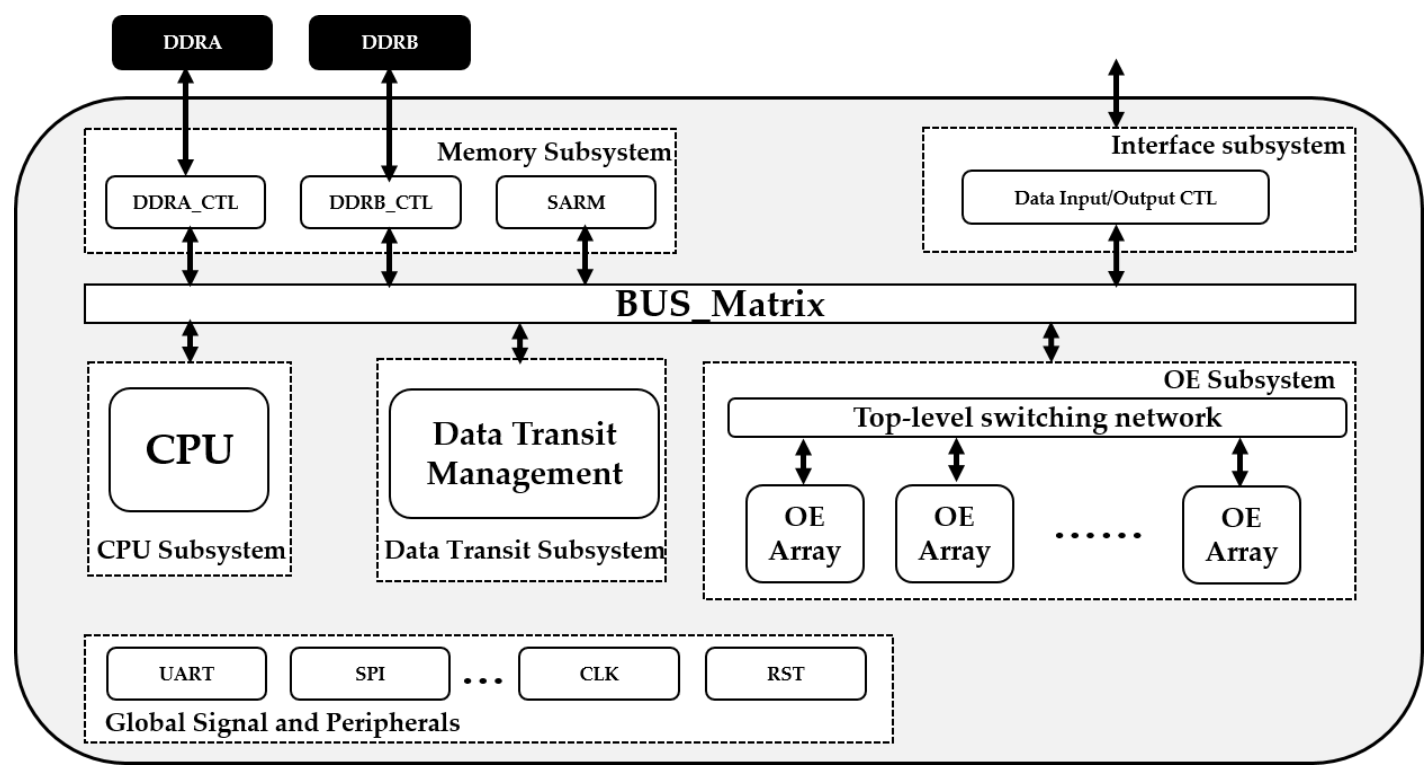

Figure 5 The framework of the integrated spaceborne SAR real-time imaging processing system.

CPU Subsystem: This subsystem mainly includes the CPU processor, which is responsible for the control of the multi-mode integrated spaceborne SAR real-time imaging processing flow and the calculation of partial SAR algorithm parameters. According to the specified imaging mode, the CPU subsystem adopts the corresponding flow control according to the multi-mode integrated spaceborne SAR real-time imaging processing flow to complete the SAR imaging processing and is also responsible for the calculation of some algorithm parameters.

Data Transit Subsystem: This subsystem is mainly responsible for moving the data stored by address, converting it into a data stream, and sending it to the computing engine subsystem. Data transit management is the core module, provides programmable addressing, supports flexible storage access, and meets the needs of different algorithm storage accesses. It also supports multiple instruction parsing and internal design instruction queuing, can achieve efficient data handling, supports SoC buses with different types of low delayed access, and provides support for parsing instruction speed controllability. With the SoC bus-to-operator sub-bus conversion function, it supports sending and receiving channels to work in parallel. The data transfer subsystem assists the processing system in completing the imaging process by taking data from the computing engine subsystem under the control of the CPU subsystem.

Operator engine sub-system: The operating engine subsystem is designed with a three-layer switching network, as shown in Figure 6. In addition to the original data stream input and resulting data stream output, the top-level exchange network is also responsible for the data interaction between the factor calculation part and the FFT-multiplication part. The FFTmultiplication part alone occupies a secondary switching network. The design described in this paper chosen two parallel FFT modules, which can flexibly configure the structure and sequence of complex multiplication and FFT pipeline work with instructions to improve the system 
reconfiguration performance. Because CS factor operation is relatively complex, multiple secondary networks are mounted in parallel on the top-level network, and the multiple parts of the above-mentioned factor are matched for parallel calculation. Simultaneously, to reduce the data exchange pressure of the secondary network, each secondary network has mounted a threelevel network to share data operation and exchange pressure. The secondary network and tertiary network constitute the same network; each network is configured with four different functions of OE computing units. The system can configure each OE to work on the pipeline through instructions, thereby realizing the efficient calculation of CS factors. Summarizing, with the configuration of the engine itself and the bus routing address, the sub-system of the arithmetic engine can realize the calculation of different formulas by different combinations of data flow and solve the compatibility problem of different calculation formula factors. The switch network designed in this paper is based on the general AXI4 architecture.

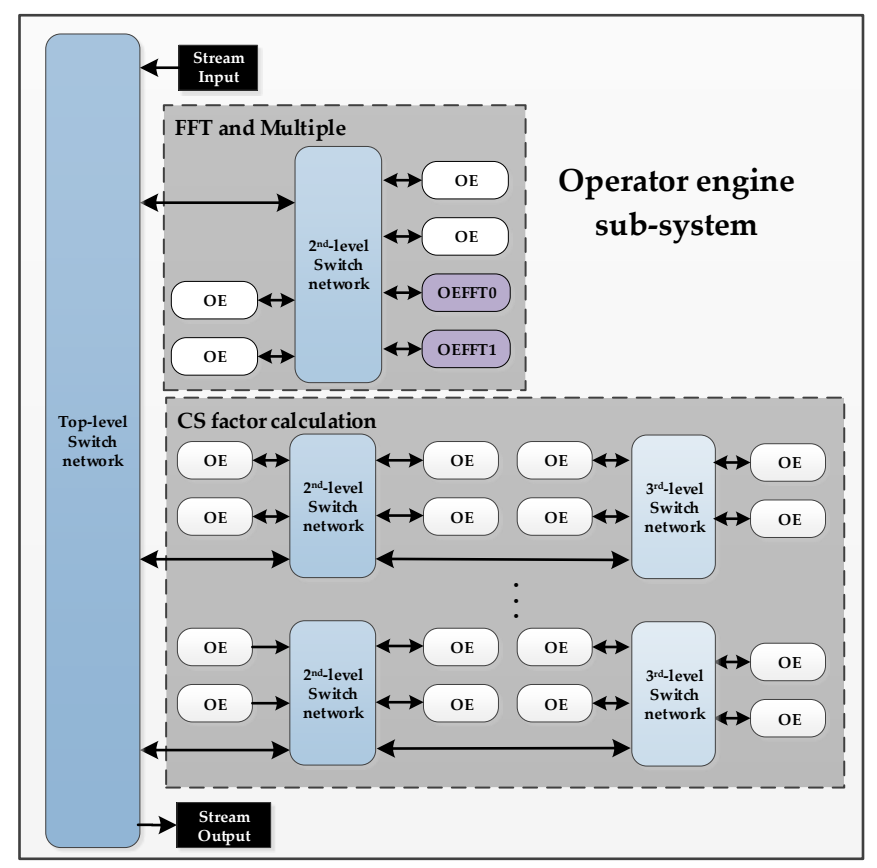

Figure 6 The architecture diagram of operator engine sub-system

Memory Subsystem: This subsystem is mainly composed of an external DDR controller and an internal SRAM controller and is responsible for the original image and intermediate data buffer in the SAR imaging process. To balance the reading and writing efficiency of SAR data in DDR storage, the literature[29] description method can be used to meet the requirements of balanced reading and writing efficiency and retain the basic data access mode of DDR memory.

Interface Subsystem: This subsystem mainly realizes high-speed input and output control of original echo data and supports serial or parallel bus data interfaces.

Global Signal and Peripherals: This is mainly composed of clock, reset, PAD control and some general peripheral interface controllers. This is mainly responsible for dealing with the internal global logic and peripheral control of the system.

The whole process is as follows: the Data Transit Subsystem is responsible for the raw input data and calculation results of data moves. Before the start of the operation, the CPU Subsystem configuration first takes all operation instructions and distributes them to each engine and subsystem from the DDR memory storage to retrieve the data and put the data into a data stream into to the Operator engine in the sub-system. After the data flow is calculated by the first $\mathrm{OE}$ engine, it will flow to the second OE according to its configured destination address, the arithmetic process flow, etc. Finally, the calculated results will be stored into DDR memory through the Memory sub-system. 


\section{Realization of the ASIC-based Accelerating Platform}

\subsection{Implementation of the Customized SAR imaging processor and the measurement system}

According to the above analysis, a prototype SAR imaging chip was implemented in a 65 $n m$ SMIC technology, as shown in Figure 7. The chip contains 5 parts as follows:

Computation logic part: The largest and most important part of the chip. The logic resource adopts a grid wiring method and contains an ARM soft core as the above-mentioned CPU part.

On-chip memory logic: This is distributed on the periphery of the Computation logic part and serves as the internal cache of the chips, with a total capacity of $20 \mathrm{MB}$.

DDR logic: This connects to the external DDR3 chip and realizes data access to the DDR3 memory. The proposed chips support three external groups, where each group has 8 GB of storage capacity of DDR3 chips for parallel data access.

Serdes PHY: This is mainly responsible for high-speed raw data input and result output. The chip supports 2 external groups of 4x SERDES interfaces with a lane rate of $3.125 \mathrm{Gbps}$.

GPIO: This is the low-frequency input/output interfaces shown in the yellow part of the microphotograph, distributed at the periphery of the chip and used for extending control and interrupt application interfaces.

Table 4 summarizes the main characteristics of the chip. A logic source of $2005.8 \times 10^{4}$ gates is integrated into a $35 \mathrm{~mm} \times 35 \mathrm{~mm}$ area, and the total power consumption is only $6.147 \mathrm{~W} @ 200$ $\mathrm{MHz}$ in standard mode. In addition, we also test the working power in low-temperature, highvoltage mode and high-temperature, low-voltage mode to suit space application environments.
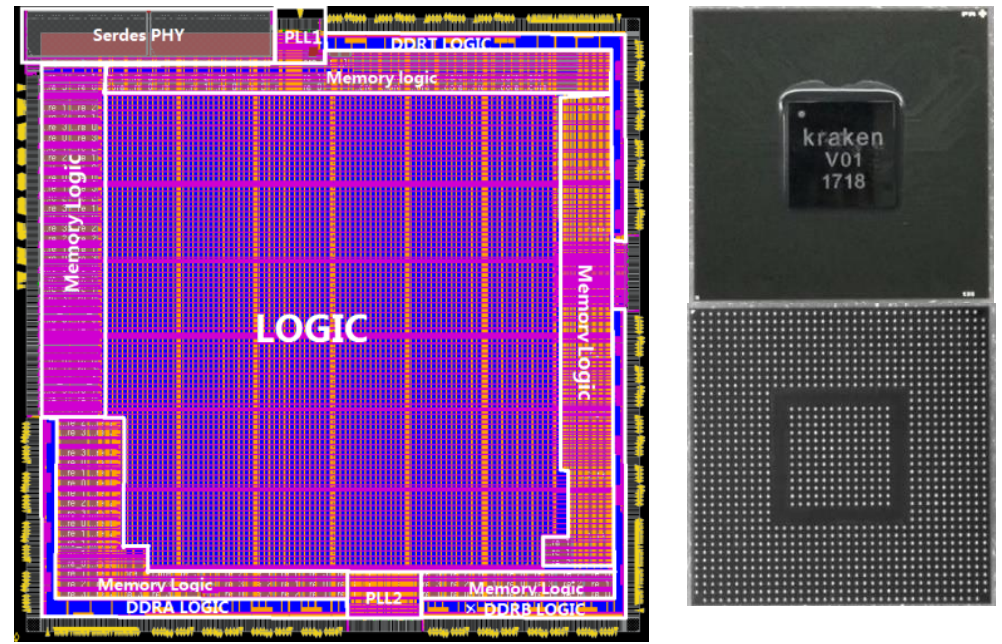

Figure 7 The microphotograph of the prototype SAR imaging chip.

Table 4 Chip characteristics

\begin{tabular}{cc}
\hline Parameters & Value \\
\hline Technology & $65 \mathrm{~nm}$ SMIC \\
Package & Flip-Chip 1245 \\
Chip area & $35 \mathrm{~mm} \times 35 \mathrm{~mm}$ \\
Clock frequency & $200 \mathrm{MHz}$ \\
Logic source & $2005.8 \times 10^{4}(\mathrm{Gate})$ \\
& Low temperature, high voltage mode \\
& $\left(-40^{\circ} \mathrm{C} ; 1.32 \mathrm{~V}\right): 4.565 \mathrm{~W}$ \\
Power & Standard mode \\
& $\left(25^{\circ} \mathrm{C} ; 1.2 \mathrm{~V}\right): 6.147 \mathrm{~W}$ \\
& High temperature, low voltage mode $\quad\left(125^{\circ} \mathrm{C}\right.$ \\
\end{tabular}




$\begin{array}{cc}\text { On-chip memory } & 20 \mathrm{Mb} \\ \text { Weight } & 50 \mathrm{~g} \\ \text { Pin Count } & 506 \\ \text { IO supply voltage } & \text { Serdes: } 2.5 \mathrm{~V}, \text { GPIO: } 3.3 \mathrm{~V} \\ \text { Internal voltage } & 1.2 \mathrm{~V} \\ \text { IR-drop } & 3.1 \%\end{array}$

\subsection{Implementation of the measurement system}

The prototype chip is packaged on PCB boards, shown in Figure 8. The chip is mainly composed of one ASIC chip, three groups of DDR3 SDRAM (DDRA and DDRB for raw data and DDRT for the CS factors) and many other high- and low-speed interface peripherals. Based on the chip test platform, we set up the measurement system, which includes the power, the simulator of the spaceborne SAR, the chip test platform and the corresponding display platform. The simulator, consisted by Xilinx ZYNQ board, is responsible for the raw data playback and transmits to the chip test platform via a high-speed interface. Finally, the imaging result can be shown through the chip function display.
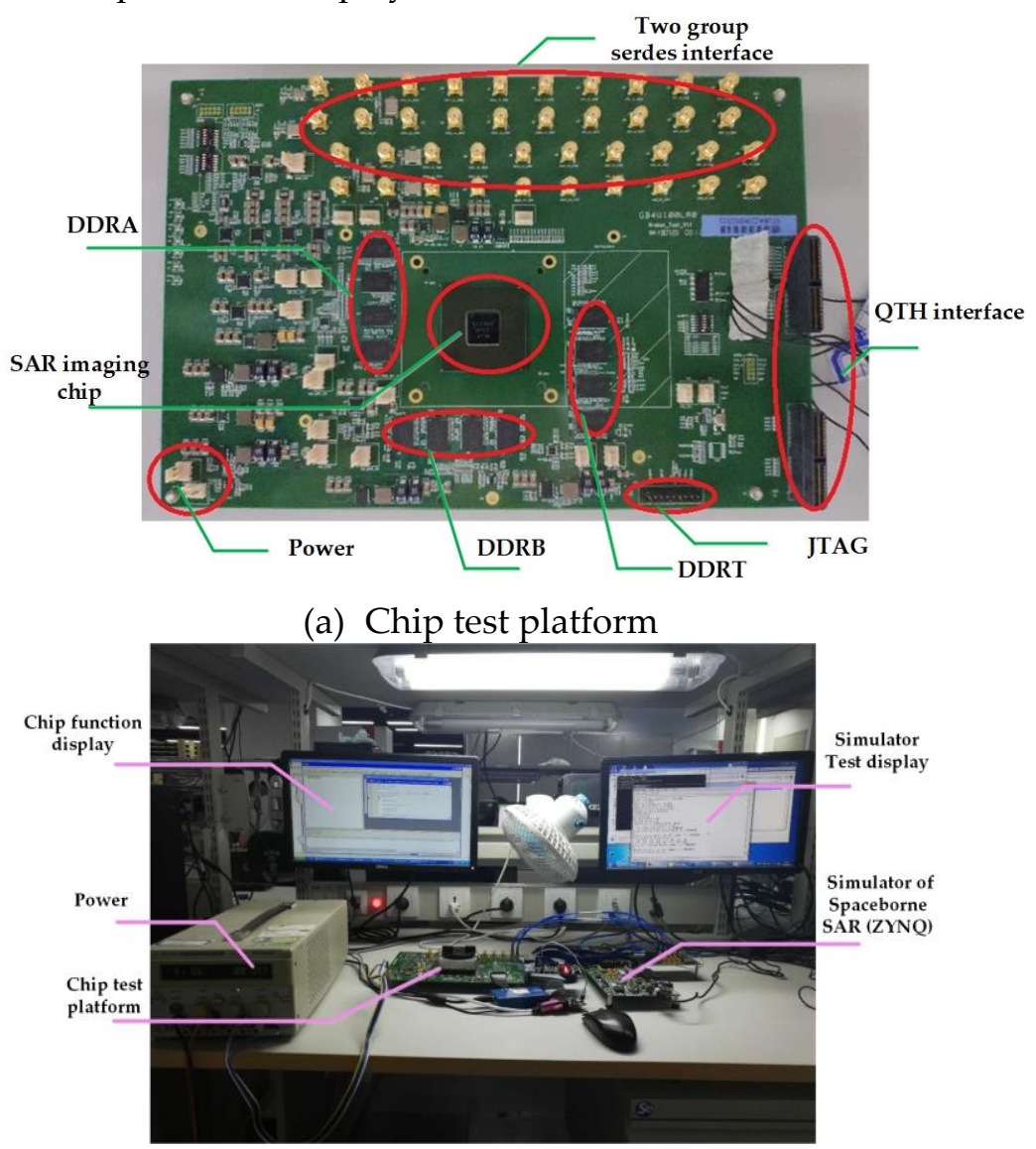

(b) The measurement system.

Figure 8 The measurement environment

\subsection{Experiment, performance and comparison}

The proposed chip based on the double-operation engine reconfigurable SoC architecture can execute multiple modes in a flexible manner. Imaging quality and time performance are the two key indicators of on-board SAR systems. The raw data with different modes that we chose to test the performance of the chip on are sourced from the Chinese Gaofen-3 satellite and which include $16,384 \times 16,384$ points for stripmap mode, $65,536 \times 8192$ points for multi- 
channel stripmap mode and $32,768 \times 4096$ points as multi-channel scan mode data. The actual scene imaging result of the measurement system is shown in Figure 9.

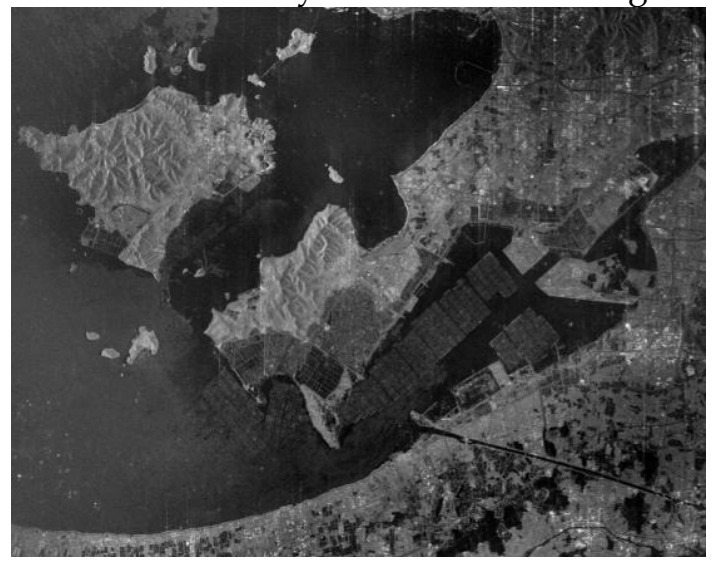

(a) Stripe mode imaging result

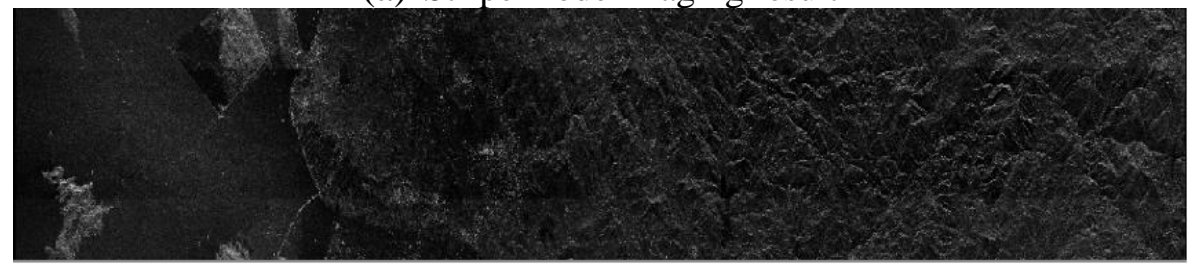

(b) Two-channel stripe mode imaging result

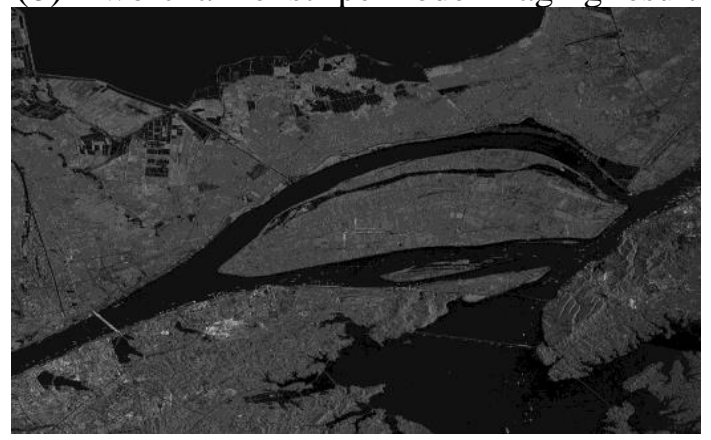

(c) Two-channel scan mode imaging result

Figure 9 Imaging result

By recording the numbers of clock cycles, we find that it takes $9.2 \mathrm{~s}, 50.6 \mathrm{~s}$ and $7.4 \mathrm{~s}$ for stripmap, multi-channel stripmap and multi-channel scan mode, respectively, and $6.9 \mathrm{~W}$ for the system hardware to process the SAR raw data. Table 7 shows a comparison with previous works. By comparison the e main advantage of chip is that it can realize multi-mode imaging. In addition, the time and power consumptions are less than those of the related design described in [28] and [29] because the proposed the ASIC has higher integration. Compared with references[2], [33], [34] , [26] and[35] , considering the data granularity processed, the proposed system shows advantages in both processing time and power consumption. Although [12] takes only 2.8 s to process SAR raw data with $32,768 \times 32,768$ granularity, the large power consumption of the GPU is unacceptable with respect to the strict spaceborne on-board real-time processing requirements.

Table 5 Comparison with previous works

\begin{tabular}{|c|c|c|c|c|c|c|c|}
\hline Works & Year & Schemes & Mode & $\begin{array}{c}\text { Data } \\
\text { granularity }\end{array}$ & $\begin{array}{l}\text { Working } \\
\text { frequency }\end{array}$ & $\begin{array}{c}\text { Power } \\
\text { consumption }\end{array}$ & $\begin{array}{c}\text { Processing } \\
\text { time }\end{array}$ \\
\hline & & & stripmap & $16,384 \times 16,384$ & & & $9.2 \mathrm{~s}$ \\
\hline Proposed & 2018 & ASIC & $\begin{array}{c}\text { Dual-channel } \\
\text { stripmap }\end{array}$ & $65536 \times 8192$ & $200 \mathrm{MHZ}$ & $6.9 \mathrm{~W}$ & $50.6 \mathrm{~s}$ \\
\hline
\end{tabular}




\begin{tabular}{|c|c|c|c|c|c|c|c|}
\hline & & & $\begin{array}{l}\text { Dual-channel } \\
\text { scan }\end{array}$ & $32768 \times 4096$ & & & $7.4 \mathrm{~s}$ \\
\hline [29] & 2017 & FPGA & stripmap & $16,384 \times 16,384$ & $100 \mathrm{MHZ}$ & $17 \mathrm{~W}$ & $10.6 \mathrm{~s}$ \\
\hline [28] & 2017 & FPGA+ASIC & Stripmap & $16,384 \times 16,384$ & $100 \mathrm{MHZ}$ & $21 \mathrm{~W}$ & $12.1 \mathrm{~s}$ \\
\hline$[2]$ & 2016 & $\begin{array}{c}\text { FPGA+ } \\
\text { Microprocess }\end{array}$ & l & $6472 \times 3328$ & / & $68 \mathrm{~W}$ & $8 \mathrm{~s}$ \\
\hline [12] & 2016 & CPU+GPU & Stripmap & $32,768 \times 32,768$ & l & $>330 \mathrm{~W}$ & $2.8 \mathrm{~s}$ \\
\hline [33] & 2015 & Multi-DSP & / & $4096 \times 4096$ & $100 \mathrm{MHZ}$ & / & $2.178 \mathrm{~s}$ \\
\hline [34] & 2012 & CPU+ASIC & / & $1024 \times 1024$ & $100 \mathrm{MHz}$ & $10 \mathrm{~W}$ & / \\
\hline [26] & 2008 & Multi-DSP & / & $4096 \times 4096$ & $100 \mathrm{MHZ}$ & $35 \mathrm{~W}$ & $13 \mathrm{~s}$ \\
\hline [35] & 1998 & ASIC & / & $1020 \times 200$ & $10 \mathrm{MHz}$ & $2 \mathrm{~W}$ & / \\
\hline
\end{tabular}

\section{Conclusions}

In this paper, to perform on-board multi-mode SAR imaging processing tasks, a float-point imaging chip based on a double-operation engine and multilayer switching network reconfigurable SoC ASIC architecture is proposed. With an efficient mapping methodology, the SAR imaging operations can be decomposed into 8 types of double-operation engines, and the full procedure can be implemented by a data-stream-driven multilayer switching network. The efficient architecture achieves real-time performance with low power consumption. A singlechip board requires $20.12 \mathrm{~s}, 43.23 \mathrm{~s}$ and $10.05 \mathrm{~s}$ on a stripmap with $16,384 \times 16,384$ granularity, a multi-channel stripmap of $65.536 \times 8192$ granularity and a multi-channel scan mode of $32,768 \times 4096$ granularity, respectively, as well as $6.9 \mathrm{~W}$ for the system hardware to process the SAR raw data. The indicators of the chip, such as weight, volume, and power, can satisfy the requirements imposed by spaceborne SAR imaging processing. With the development of antiradiation reinforcement technology and system fault-tolerant technology, the proposed framework is found to be expandable and feasible for potential spaceborne SAR imaging processing.

Acknowledgments: This work was supported by the National Natural Science Foundation of China under Grant 91438203, the Chang Jiang Scholars Program under Grant T2012122 and the Hundred Leading Talent Project of Beijing Science and Technology under Grant Z141101001514005.

Author Contributions:, Zhu Yang and Bingyi Li conceived and designed the framework. Zhu Yang, Bingyi Li and Liang Chen applied the mapping technology and analysed the data. Zhu Yang and Yizhuang Xie debugged the system hardware. Zhu Yang and Bingyi Li wrote the paper. Teng Long and He Chen reviewed papers and submitted comments.

Conflicts of Interest: The authors declare no conflicts of interest.

\section{References}

1. Franceschetti, G. and R. Lanari. Synthetic Aperture Radar Processing. in Geoscience and Remote Sensing Symposium, 1994. Igarss '94. Surface and Atmospheric Remote Sensing: Technologies, Data Analysis and Interpretation., International. 1999.

2. Lou, Y., et al., Onboard Radar Processor Development for Rapid Response to Natural Hazards. IEEE Journal of Selected Topics in Applied Earth Observations and Remote Sensing, 2016. 9(6): p. 2770-2776.

3. Hirose, A., et al., Foreword to the Special Issue on Advances in SAR and Radar Technology. Selected Topics in Applied Earth Observations \& Remote Sensing IEEE Journal of, 2015. 8(8): p. 37483750. 
4. Gierull, C.H. and P.W. Vachon, Foreword to the Special Issue on Multichannel Space-Based SAR. Selected Topics in Applied Earth Observations \& Remote Sensing IEEE Journal of, 2015. 8(11): p. 4995-4997.

5. Tralli, D.M., et al., Satellite remote sensing of earthquake, volcano, flood, landslide and coastal inundation hazards. Isprs Journal of Photogrammetry \& Remote Sensing, 2005. 59(4): p. 185198.

6. $\quad$ Percivall, G.S., et al., Improving Disaster Management Using Earth Observations - GEOSS and CEOS Activities. IEEE Journal of Selected Topics in Applied Earth Observations \& Remote Sensing, 2013. 6(3): p. 1368-1375.

7. Joyce, K.E., et al., A review of the status of satellite remote sensing and image processing techniques for mapping natural hazards and disasters. Progress in Physical Geography, 2009. 33(2): p. $183-$ 207.

8. Copernicus: Sentinel-1 - The SAR Imaging Constellation for Land and Ocean Services. [cited 2017 23th Mar.]; Available from: https://directory.eoportal.org/web/eoportal/satellite-missions/cmissions/copernicus-sentinel-1.

9. TDX (TanDEM-X: TerraSAR-X add-on for Digital Elevation Measurement). [cited 201723 th Mar.]; Available from: https://directory.eoportal.org/web/eoportal/satellitemissions/t/tandem-x.

10. ALOS-2 (Advanced Land Observing Satellite-2; SAR mission) / Daichi-2. [cited 2017 23th Mar.]; Available from: https://directory.eoportal.org/web/eoportal/satellite-missions/a/alos-2.

11. Zhang, F., et al., Multiple Mode SAR Raw Data Simulation and Parallel Acceleration for Gaofen-3 Mission. 2018. PP(99): p. 1-12.

12. Zhang, F., et al., Accelerating Spaceborne SAR Imaging Using Multiple CPU/GPU Deep Collaborative Computing. Sensors, 2016. 16(4).

13. Wong, F.W., T.S.J.I.T.o.G. Yeo, and R. Sensing, New applications of nonlinear chirp scaling in SAR data processing. 2002. 39(5): p. 946-953.

14. Moreira, A., et al., Extended chirp scaling algorithm for air-and spaceborne SAR data processing in stripmap and ScanSAR imaging modes. 1996. 34(5): p. 1123-1136.

15. Showman, G.A., Stripmap SAR. 2012: IET Digital Library. 259-335.

16. Proctor, P.J.A.W. and S. Technology, Spotlight sar. 1997.

17. Moreira, A. and J. Mittermayer, Method for azimuth scaling for SAR-data and high precision processor for two-dimensional processing of scan-SAR data. 1998, EP.

18. Ender, J.H.G.J.P.E., Detection and estimation of moving target signals by multi-channel SAR. 1996. 50(2): p. 150-156.

19. Meta, A., et al., TOPS Imaging With TerraSAR-X: Mode Design and Performance Analysis. 2010. 48(2): p. 759-769.

20. Song, W.S., E.J. Baranoski, and D.R. Martinez. One trillion operations per second on-board VLSI signal processor for Discoverer II space based radar. in Aerospace Conference Proceedings. 2000.

21. Langemeyer, S., et al. A compact and flexible multi-DSP system for real-time SAR applications. in Geoscience and Remote Sensing Symposium, 2003. IGARSS '03. Proceedings. 2003 IEEE International. 2004. 
22. Wai-Chi, F. and M.Y. Jin. On board processor development for NASA's spaceborne imaging radar with VLSI system-on-chip technology. in 2004 IEEE International Symposium on Circuits and Systems (IEEE Cat. No.04CH37512). 2004.

23. Le, C., et al., Onboard FPGA-based SAR processing for future spaceborne systems. Proceedings of the Ieee 2004 Radar Conference, 2004: p. 15-20.

24. Fang, W.C., C. Le, and S. Taft. On-board fault-tolerant SAR processor for spaceborne imaging radar systems. in IEEE International Symposium on Circuits and Systems. 2005.

25. Greco, J., et al., Hardware/software Interface for High-performance Space Computing with FPGA Coprocessors. 2006: p. 1-10.

26. Desai, N.M., et al. Near Real Time SAR Processors for ISRO'S Multi-Mode RISAT-I and DMSAR. in 7th European Conference on Synthetic Aperture Radar. 2008.

27. Pfitzner, M., et al. FPGA based architecture for real-time SAR processing with integrated motion compensation. in Synthetic Aperture Radar. 2013.

28. Yang, C., et al., A Spaceborne Synthetic Aperture Radar Partial Fixed-Point Imaging System Using a Field-Programmable Gate Array-Application-Specific Integrated Circuit Hybrid Heterogeneous Parallel Acceleration Technique. Sensors, 2017. 17(7).

29. Li, B., et al., Real-Time Spaceborne Synthetic Aperture Radar Float-Point Imaging System Using Optimized Mapping Methodology and a Multi-Node Parallel Accelerating Technique. 2018. 18(3): p. 725.

30. Yang, Z., et al., Implementation of CS Coefficient Calculation Based on Dual-Operator Engines in Multimode Spaceborne SAR Imaging Systems. 2017. 54(3).

31. Raney, R.K., et al., Precision SAR processing using chirp scaling. IEEE Transactions on Geoscience \& Remote Sensing, 1994. 32(4): p. 786-799.

32. Ding, Data processing of multi-channel space-borne SAR. 2009(551): p. 1-4.

33. Gu, C.F., et al., Multi-Core DSP Based Parallel Architecture for FMCW SAR Real-Time Imaging. Radioengineering, 2015. 24(4): p. 1084-1090.

34. Bierens, L. and B.J. Vollmuller. On-board Payload Data Processor (OPDP) and its application in advanced multi-mode, multi-spectral and interferometric satellite SAR instruments. in EUSAR 2012; 9th European Conference on Synthetic Aperture Radar. 2012.

35. Franceschetti, G., et al. A VLSI architecture for real time processing of one-bit coded SAR signals. in Ursi International Symposium on Signals, Systems, and Electronics. 1998. 